\title{
MaLT - combined motor and language therapy tool for brain injury patients using Kinect
}

Article

Accepted Version

Wairagkar, M., McCrindle, R., Robson, H., Meteyard, L., Sperrin, M., Smith, A. and Pugh, M. (2017) MaLT - combined motor and language therapy tool for brain injury patients using Kinect. Methods of Information in Medicine, 56 (2). pp. 127137. ISSN 2511-705X doi: https://doi.org/10.3414/ME16-020015 Available at https://centaur.reading.ac.uk/69287/

It is advisable to refer to the publisher's version if you intend to cite from the work. See Guidance on citing.

To link to this article DOI: http://dx.doi.org/10.3414/ME16-02-0015

Publisher: Schattauer Publishers

All outputs in CentAUR are protected by Intellectual Property Rights law, including copyright law. Copyright and IPR is retained by the creators or other copyright holders. Terms and conditions for use of this material are defined in the End User Agreement.

www.reading.ac.uk/centaur 
Central Archive at the University of Reading

Reading's research outputs online 
Published paper http://dx.doi.org/10.3414/ME16-02-0015

Journal: Methods of Information in Medicine 56(2)

Title: MaLT - Combined guage Therapy Tool for Brain Injury Patients Using Kinect*

Maitreyee Wairagkar ${ }^{1}$; Rachel McCrindle ${ }^{1}$; Holly Robson ${ }^{2}$; Lotte Meteyard ${ }^{2}$;

Malcom Sperrin 3 ; Andy Smith ${ }^{4}$; Moyra Pugh 5

1Biomedical Engineering, School of Biological Sciences, University of Reading,

Reading, UK;

2School of Psychology and Clinical Language Sciences, University of Reading, Reading, UK;

3Medical Physics, Royal Berkshire Hospital Reading, Reading, UK;

${ }^{4}$ Clinical Engineering, Royal Berkshire Hospital Reading, Reading, UK;

5Physiotherapy \& Occupational Therapy, Royal Berkshire Hospital Reading,

Reading, UK

Correspondence to:

Maitreyee Wairagkar Biomedical Engineering School of Biological Sciences University of Reading Whiteknights Reading RG6 6AY UK

E-mail: m.n.wairagkar@pgr.reading.ac.uk

Keywords

Multidisciplinary, motor rehabilitation, language rehabilitation, stroke, brain injury, games based therapy, Kinect 


\section{Summary}

Background: The functional connectivity and structural proximity of elements of the language and motor systems result in frequent co-morbidity post brain injury. Although rehabilitation services are becoming increasingly multidisciplinary and "integrated", treatment for language and motor functions often occurs in isolation. Thus, behavioural therapies which promote neural reorganisation do not reflect the high intersystem connectivity of the neurologically intact brain. As such, there is a pressing need for rehabilitation tools which better reflect and target the impaired cognitive networks.

Objectives: The objective of this research is to develop a combined high dosage therapy tool for language and motor rehabilitation. The rehabilitation therapy tool developed, MaLT (Motor and Language Therapy), comprises a suite of computer games targeting both language and motor therapy that use the Kinect sensor as an interaction device. The games developed are intended for use in the home environment over prolonged periods of time. In order to track patients' engagement with the games and their rehabilitation progress, the game records patient performance data for the therapist to interrogate.

Methods: MaLT incorporates Kinect-based games, a database of objects and language parameters, and a reporting tool for therapists. Games have been developed that target four major language therapy tasks involving single word comprehension, initial phoneme identification, rhyme identification and a naming task. These tasks have 8 levels each increasing in difficulty. A database of 750 objects is used to programmatically generate appropriate questions for the game, providing both targeted therapy and unique gameplay every time. The design of the games has been informed by therapists and by discussions with a Public Patient Involvement (PPI) group.

Results: Pilot MaLT trials have been conducted with three stroke survivors for the duration of 6 to 8 weeks. Patients' performance is monitored through MaLT's reporting facility presented as graphs plotted from patient game data. Performance indicators include reaction time, accuracy, number of incorrect responses and hand use. The resultant games have also been tested by the PPI with a positive response and further suggestions for future modifications made.

Conclusion: MaLT provides a tool that innovatively combines motor and language therapy for high dosage rehabilitation in the home. It has demonstrated that motion sensor technology can be successfully combined with a language therapy task to target both upper limb and linguistic impairment in patients following brain injury. The initial studies on stroke survivors have demonstrated that the combined therapy approach is viable and the outputs of this study will inform planned larger scale future trials. 


\section{Introduction}

Whilst improved acute medical care has increased survival rates following an acquired brain injury $(A B I)$, it has led to a growing prevalence of individuals with longterm rehabilitation needs. Within the UK alone over $1.2 \mathrm{~m}$ individuals live with the long-term consequences of stroke [1] and 120,000 with the effects of traumatic brain injury (TBI). Within this population language impairments and motor impairments are common. Up to $77 \%$ of acute stage stroke survivors and up to $45 \%$ of chronic TBI survivors can experience difficulties in using upper limbs [2,3] and approximately one third of individuals with stroke and TBI suffer from language impairments $[4,5]$.

Motor and language impairments have long-term implications for individuals' functional independence, social contact, self-esteem and mental health $[6,7,8]$. Neurobiologically, the language and motor networks lie in close proximity $[9,10]$ and are, for the most part, perfused by the same artery [11]. This makes these systems vulnerable to the same neurological incident, resulting in frequent co-morbidity. In the typical brain, the motor and language systems are not only structurally proximal but also functionally interconnected [12] and there is preliminary evidence that coactivation of motor and language regions may be associated with recovery from acquired brain injury $(A B I)[13,14]$. Individuals with stroke and TBI can show significant improvement from the same language treatments [15] and the same motor treatments [16]. There is also evidence that language treatments improve when combined with higher physical activity (standing rather than sitting) [17]. However, to our knowledge, motor and speech rehabilitation goals have never been integrated into the same treatment. Given that impairment based therapy attempts to improve neural functioning, a therapeutic approach which reflects the interconnectivity of cognitive systems in the typical brain might lead to increased long-term success over therapy which addresses cognitive systems in isolation.

Dosage is another factor known to influence therapy success. Significant correlations have been identified between hours in therapy and outcome, with "successful" programmes associated with approximately 100 hours of therapy time [18]. It is not possible to provide such high intensity therapy with the traditional 1:1 model. A recent speech and language therapy efficacy study showed that patient and speechlanguage therapist contact time averages only 18 hours in the first 4 months post onset [19]. As such, need for intervention greatly exceeds therapy time with many individuals. The need to develop cost-effective and efficient therapeutic tools which allow individuals to engage in long-term home-based rehabilitation is pressing.

There currently exists a range of computer-based language programs, mechanical motor rehabilitation products such as balance boards and finger/grip strengthening devices and, since the advent of the Wii and Kinect, a number of motion sensor based systems designed to aid recovery of motor skills [e.g. 20, 21, 22]. However, as stated above, no systems currently exist which integrate these therapeutic domains. Furthermore, computer-based language rehabilitation is often static in terms of difficulty levels and capability for therapists to easily adapt it as per the patient needs. It is crucial to replicate the adaptive skills of the clinician for a therapeutic product to be accessible and useful to a wide range of impairment severity.

This project is targeting three core areas: (1) Need for more theoretically-grounded, evidence-based rehabilitation tools; (2) Need for motivating tools that allow patients to engage in time-efficient, yet still personalised, home-based rehabilitation; (3) Need for tools that promote an integrated, interdisciplinary approach to rehabilitation. 


\section{Objectives}

The overall objective of this research is to develop a high dosage combined language and motor rehabilitation tool for use with individuals with $A B I$. The key premise of the tool is to deliver the combined therapy via a suite of interactive Kinect based computer games that present language therapy questions to the patient which are answered by performing physical movement of the affected arm, thereby creating a form of exergaming.

In order to achieve this main objective, several key requirements for the tool were identified.

Provision of therapy: games had to incorporate a number of bespoke language tasks designed by speech and language therapists including levels of increasing difficulty for each task.

Usability: games developed had to be very intuitive to play and easy to set up and use such that patients did not require any special instructions or training to play.

Engaging: games had to be designed so that they were engaging and challenging for the patients without compromising on the therapy provided. To ensure unique gameplay each time a patient played the games, questions had to be generated automatically from a large database of objects whilst obeying rules for correct therapy interventions.

Feedback: the games had to provide easy to comprehend feedback and reinforcement to patients in the form of score and emoticons.

Monitoring: there had to be the facility for therapists to monitor patients' progress as they completed the different levels of game difficulty both within a session and across multiple sessions.

Flexibility: the tool had to provide the option for therapists to tailor the database and gameplay according to an individual patient's needs.

Reporting: the tool had to record all gameplay data automatically such that it could provide rich data about a patient's progress in a format that was easily accessible by the therapist.

Low cost and home environment: the tool and its games had to playable in the home at an affordable cost. This was achieved by developing a Windows based game which could be played on a computer or a laptop using a Kinect sensor.

Playability: The game should be effective, motivating and provide satisfaction and learning. It should also be easy to play along with being engaging, leading to successful language therapy.

The viability of the games and inclusion of the above requirements have been tested with stroke patients by conducting the pilot clinical trials in patients' home environments for prolonged duration across $6-8$ weeks. 


\section{Methods}

\subsection{Collaborative Development and Patient Involvement}

Service users, speech and language therapists, physiotherapists and occupational therapists were involved collaboratively in

the design of the clinical and usability aspects of the therapy. Language therapists from the School of Clinical Language Sciences, University of Reading identified the requirements for the language therapy for stroke patients and designed the therapy used in MaLT. Physiotherapists and Occupational therapists from Royal Berkshire Hospital worked collaboratively in identifying the patient requirements and motor aspect of the therapy by emphasizing the need for longer therapy dosage which is not possible in clinical settings. Human Computer Interaction, Rehabilitation experts, Cyberneticist and Software Engineers from Biomedical Engineering, University of Reading developed the games fulfilling all the objectives listed in the previous section. Thus, the experts from various fields worked collaboratively to design and develop this unique MaLT therapy game.

A Patient Public Involvement (PPI) Group was also involved throughout the development of the therapy tool. The PPI group involved three members, F a partner of a stroke survivor, $S$ and $B$, both stroke survivors who suffered the stroke 11 months prior to onset of the therapy tool development. Both $S$ and $B$ suffered from aphasia, an acquired language impairment, and upper-limb weakness. The PPI, software developer and clinical team members met for discussions and trial of the games thrice during the course of the project - before beginning the development, during the development and after completion of the therapy tool.

In addition to the requirements outlined in section 2, initial consultation with the PPI group confirmed satisfaction with the premise of the therapy and provided further core recommendations for development: (1) The need for regular breaks in the therapy was emphasised; (2) An easy game component to be included as a "warmup"; (3) Minimum decision making points through the game; (4) Capacity to set difficulty level.

\subsection{Game Development}

The MaLT (Motor and Language Therapy) tool has been developed to target language and motor therapy simultaneously by using exergaming for answering language therapy questions. MaLT has been developed in C\# and Unity Game Engine for Windows Desktop. It uses the Kinect 2 sensor to recognise the movements and gestures of the user and enables the user to interact with the objects in the game. Kinect 2 can track 25 joints on the body and create a real time wireframe or skeleton of a player's body. The skeleton of a patient's body is displayed on the screen which helps them to position themselves on the screen and observe their movements. This gives good visual feedback during the game and helps brain injury patients to self-monitor their movements and make the efforts required for any necessary improvements.

MaLT consists of a series of games targeting language therapy and the patient plays the game by performing movements of upper-limbs which in turn provides motor therapy. Patients may be standing or sitting. Motor therapy in MaLT is based on the PURR (Prescriptive Software for Use in Recovery and Rehabilitation) system [23]. Language therapy tasks have been incorporated into the game aimed at improving cognitive language tasks such as understanding words, differentiating between 
closely related words, identifying and distinguishing speech sounds (phonemes), identifying rhyming words, and retrieving words.

The games involve different language questions being displayed on the screen. Pictures of objects are presented on the screen as options to each question, with one being the target item and the rest distracters. The patient has to select the correct answer by extending their right or left arms to reach the objects on the screen. The patient is given multiple attempts to select the target item until the correct answer is selected. The patient receives both auditory and visual feedback in the form of the spoken name of the item selected and a tick or a ' $X$ ', as shown in Figure 1 (a), (b) and (c). Hearing the word spoken aloud correctly also gives positive reinforcement for relearning the words.

Each game has eight levels which increase in complexity as a function of familiarity of the target item, proximity of the distracter items and number of distracters. These eight levels have been categorised into easy (level 1-3), medium (level 4-6) and hard (level 7 and 8) categories that patients can select. During each level, up to 20 questions are presented to the patients. In order to generate appropriate questions for each task at the different levels, sets of rules have been created. The patients can select the game, the level of difficulty and number of questions in the game before beginning the game. The starting screens with different options are shown in Figure 2 (a), (b) and (c).

An XML database stores objects and their different language parameters such as number of syllables, initial phoneme, semantic category, rhyming vowel, rhyming words, and familiarity of the object. The database contains 750 objects from which questions are generated programmatically for each level of each task according to set rules.

MaLT allows performance monitoring of the cognitive language tasks as well as comparison of performance and quality of movement of the affected and normal arm.

\subsection{Language Therapy Games}

MaLT is divided into three major therapy tasks (1) single word comprehension, (2) initial phoneme identification and (3) finding rhyming words and a speaking task to reinforce word retrieval and articulation. The game uses Text to Speech software [24] to read out the question along with displaying the text on the screen. Multimodal input is designed to facilitate question comprehension.

Once the game starts, all the game controls are gesture based so that the patient does not have to shift between selecting the options on the computer with mouse and keyboard and playing the game by performing movements which are registered by the Kinect. After each level of the game, the score is displayed on the screen along with an emoticon indicating the quality of performance for instant feedback to the patients. A sad face for low performance below 45\%, happy face for medium performance up to $70 \%$ and very happy face for excellent performance above $70 \%$ are displayed. The feedback screen is shown in the Figure 2 (d). The navigation option is provided for starting a new level or going back to the main menu. An option for resuming the game is also provided.

\subsubsection{Single Word Comprehension}

The single word comprehension task requires the patient to match a written and spoken word to a picture item. The single word comprehension game is graded in 
difficulty by familiarity of item, similarity of distracters (semantic and phonological overlap) and number of distracters e.g. moon - star, jam - bread etc. The user is asked to select an object from the images presented on the screen. As the difficulty level rises, the number of distracters increases, the familiarity of the objects decreases, and semantically closer associated objects are given as options for the answer with varying phonological restrictions for increasing the challenge. An example of the single word comprehension game is shown in Figure 1 (a).

\subsubsection{Initial Phoneme}

The initial phoneme task (see Figure 1 (b)) tests the ability of the patient to associate the pictures of objects on the screen with their names and identify their initial phonemes. The question takes the form of "Find the one that starts with 's'". This task challenges the user at multiple levels, firstly, identifying the phoneme from the question, then identifying the objects on the screen and generating possible names, isolating their initial phonemes and matching to the probe phoneme. Different phonemes have different 'distinctive features' based on the configuration of the articulators required to produce the sound. The complexity of this task is increased in different levels based on these distinctive features, number of distractors and familiarity. At simpler levels target items are more familiar with greater acoustic distance between target and distracters. In the higher levels, the objects with sounds closely related are presented e.g. 'sh' and 'v', 'f ', 's..' and 'z..' (the buzzing sounds).

(a) Single word comprehension game

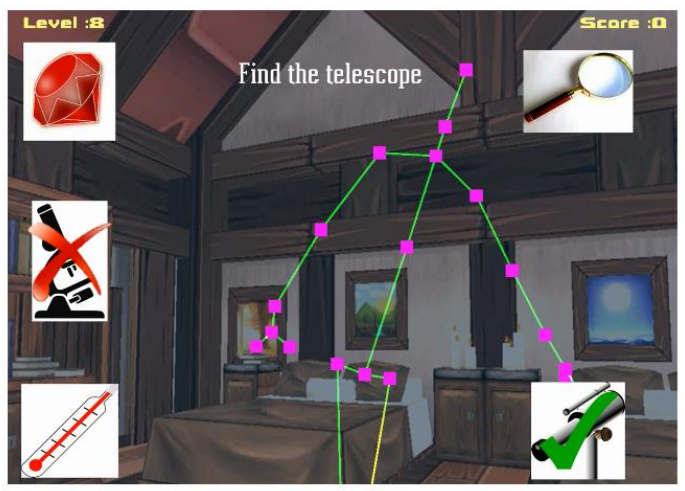

(c) Rhyming word game

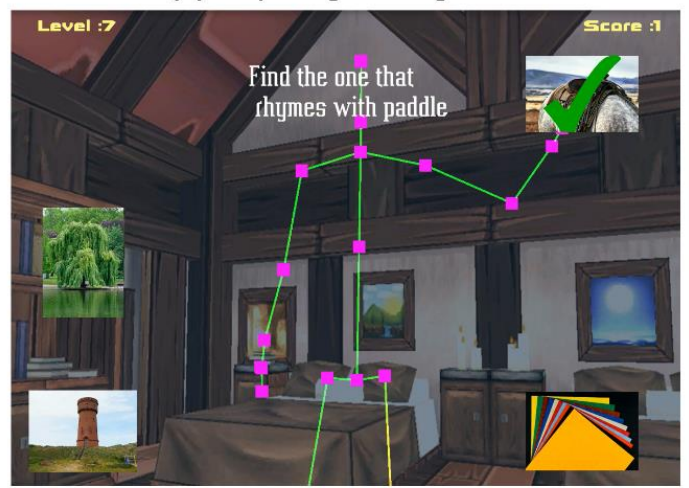

(b) Initial phoneme game

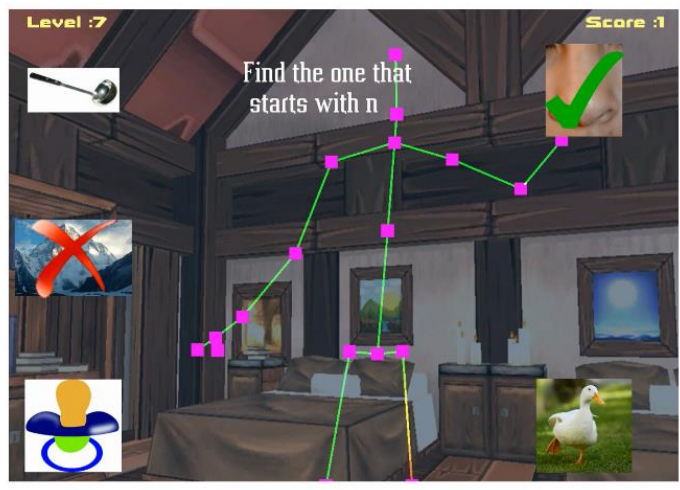

(d) Speaking task

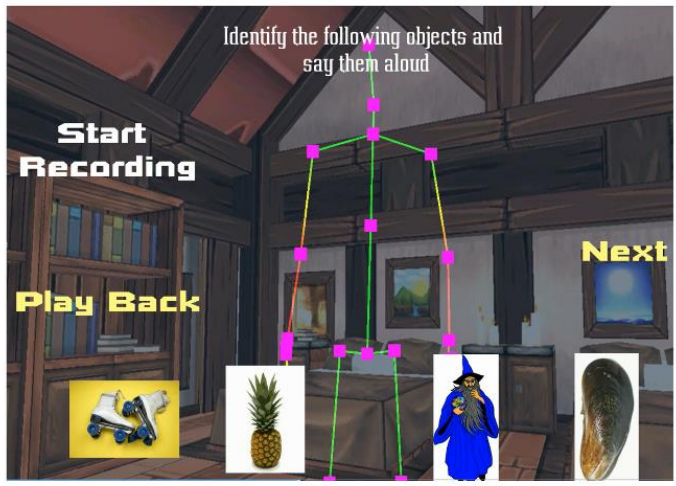

Figure 1: Screenshots of MaLT games. (a) Word comprehension game, (b) Initial phoneme identification game, (c) Rhyming word identification game, and (d) Speaking task. The selection of correct and wrong answers is indicated on the screen in screenshots (a), (b) and (c). 


\subsubsection{RhymingWord}

The rhyming task requires a similar cognitive process to the initial phoneme identification task. The patient must segment the rhyme component of the probe item, identify the range of items on the screen and generate possible names, isolate the rhyme components of those items and compare these to the probe item. This task is graded by item familiarity, phonological and semantic overlap of distracters and number of syllables. An example of the rhyming word game is shown in Figure 1 (c).

\subsubsection{SpeechTask}

After every level of the above games, an additional naming task is presented. Retrieving and producing names is a universal impairment in aphasia and this task is one of the core requests of the PPI panel. This task gives patients the opportunity to practice retrieving and producing names after priming the items in the previous game. In this task, any four target objects from the previous level are displayed on the screen. The patients have to identify these objects and say them aloud. Hovering their arms over a particular object plays the initial phoneme (initial sound) of the object, a phonemic cueing technique which is effective in facilitating name retrieval in aphasia [25]. In this task, speech can be recorded using the options given on the screen and played back to the patient for instant feedback. The recorded speech is saved for therapist's reference and progress monitoring. Patients can repeat the naming task and re-record their production to identify improvement. All the menu options for recording and playback are again accessed by movement to maintain the continuation and flow of the game. An example of the speaking game is shown in Figure $1(d)$.

\subsection{Therapy Tool Development Mechanisms}

The questions in each game are programmatically formed by querying the database according to given rules for each level thereby eliminating the need for hardcoded questions. This ensures that every time the patient plays the game, different sets of questions are generated, thus the patient will potentially play a different game every time. The large number of objects in the database helps to increase the variety of questions and avoid any repetition. This approach has also helped to generate a more flexible and adaptable programming strategy for the game. Adding more objects into the game is simply a case of updating the database without the need to change any of the game programming. This also allows therapists to alter the game according to the requirements and competence of their patients and make tailor made selections of objects for individual patients.

It is known that patients are more motivated and interested when they are given certain autonomy and opportunity to show competence. The flexibility to choose the difficulty level and the type of the game provides control to the user [26]. Also, the score with an emoticon shown at the end of each level and opportunity to hear back their recorded speech provides motivation to perform better.

Patient performance data is recorded in separate XML files for interpretation by the therapist. The details of the data that is recorded is given in the results section 4.1. An independent visualization tool has been created for observing the data that extracts and tabularizes information about the patient's performance. Therapists can then view graphs of different performance measures like accuracy, response time, 
time spent on therapy and other features relevant to the patient as specified by the therapist. A web based visualization tool allowing more specific faster analysis of patient data is being developed and will be integrated with the MaLT system in the future.

All three games were tested thoroughly after development to identify and fix any technical bugs that were evident in the system. From a therapy and playability perspective the main test performed on the games was to confirm that each of the 8 levels for all 3 games had enough items in the database to form targets and distractors to create a variety of different questions automatically following the given set of rules. The percentage of different items from the database seen by the participants during the course of the pilot was analysed from the patients recorded data and is reported in the results section.

\subsection{Clinical Trials with Stroke Survivors}

\subsubsection{Participants}

A pilot study to investigate adherence to the therapy programme was completed with three stroke survivors: IB, RH, RB (ages 45 to 62 years) with right side weakness. All patients were in the chronic phase of their stroke, over a year since onset and suffered from speech production impairments characterised by difficulties with retrieving and articulating words. All patients had some impairment of phonological analysis. One patient (RB) had additional difficulties comprehending spoken language. NHS ethical approval for the study involving human patients was obtained. All the patients gave their written informed consent prior to the pilot testing using aphasia friendly modified information sheets and consent forms.

\subsubsection{Experimental Protocol}

Patients were given the MaLT system to use for up to 10 weeks and were encouraged to use the system as much as possible. The patients were given a single training session at the start of the programme. The patients were provided with the MaLT system which included a laptop with pre-installed MaLT and Kinect sensor. Each patient was shown how to use the MaLT system and then observed setting up and interacting with the software with further support provided if needed. Patients were provided with contact details of a researcher for technical support if needed. Researchers periodically checked with the patients throughout the programme to confirm they were not having technical difficulties using the software.

The patients were given the option to play standing or seated in front of the Kinect for each session. In each session, patients played the games of their choice in any order. The duration of each session was not fixed. The data of their trials was stored automatically in separate files for each day. 
(a) Main screen

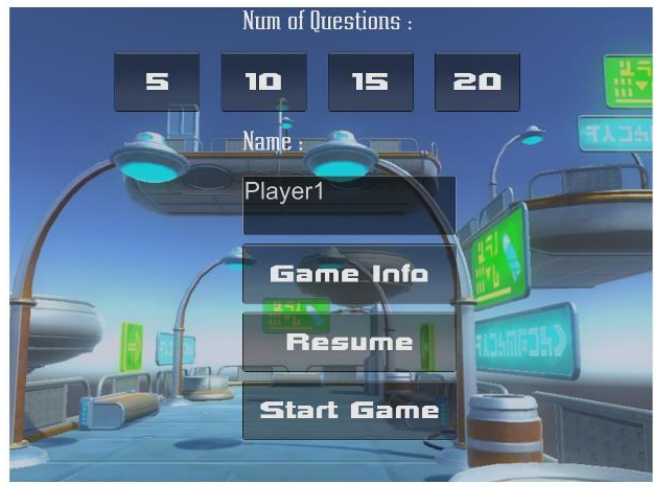

(c) Difficulty level selection screen

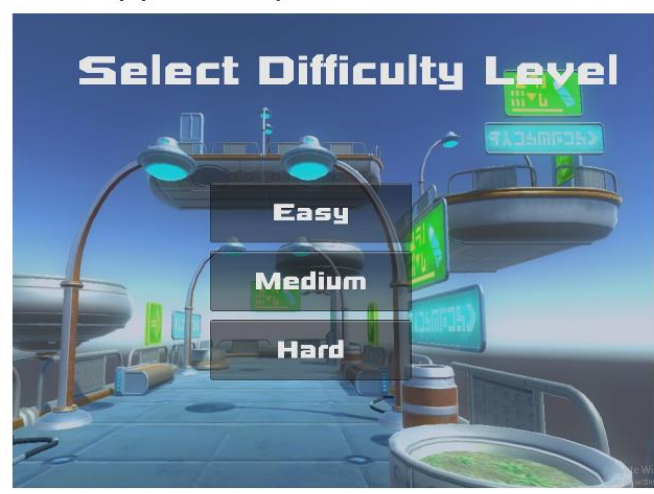

(b) Game selection screen

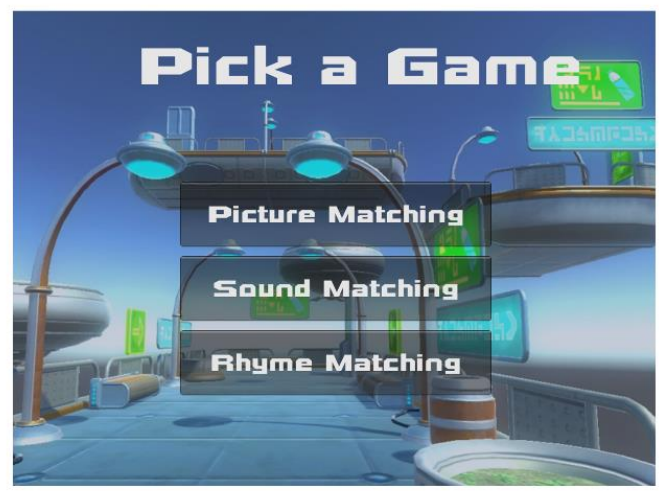

(d) End of game feedback screen

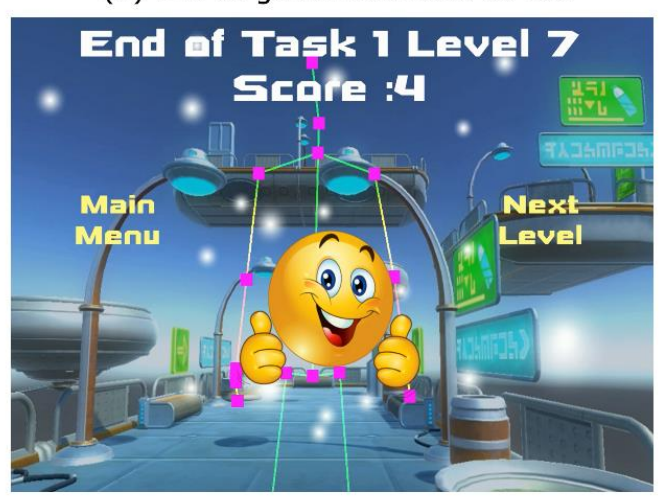

Figure 2: Screenshots of MaLT setup screens and Feedback screen. (a) Main screen to select number of questions, options to play a new game or resume game, (b) Game selection screen to select one of the three MaLT games, (c) Screen to select the difficulty level of the game, and (d) Feedback screen showing score after the game.

\subsubsection{Preliminary Analysis of the Pilot Data}

For preliminary data analysis, three measures of engagement and therapy structure have been examined. We looked at number of trials completed during the course of therapy for all the patients, time spent playing each of the therapy games and number of attempts for getting the correct answer for each game and level. Moreover, the average time statistics for answering the question was recorded for the all the patients. The results are detailed in the results section 4.3. 


\section{Results}

\subsection{MaLT Data Recording}

The game data is stored automatically during the game which can be viewed by therapists to evaluate patient progress. The game data of a patient is stored in a separate file for each day. It records details of the task, level of the question, target to be identified and distracters presented on the screen for each question. It also saves the response time in seconds and number of attempts taken to select the correct answer. The position of the target on the screen is saved along with the hand used to select the target. All this information gives a complete profile of a patient's performance. Information related to the target, distracters and number of attempts can be used to assess the progress of language therapy; whilst factors such as the response time, hand used to select the target and target position on the screen can be used to assess the progress of motor therapy. The progress of the patient can be monitored over time by the therapist by comparing plots of response times and problem areas can be identified, for example, if the patient takes more time to reach the target displayed on a particular position on the

screen, if the patient prefers the use of one hand over the other, or if patient finds it difficult to answer a question for a particular task and level. Monitoring this data will help the therapist to identify specific problem areas and change the therapy accordingly. Additional rich data can be used for further detailed interpretation of the patients' performance in different language and motor tasks. The joint positions of both the hands, elbows, shoulders and neck are recorded continuously during the trial for future analysis of the trajectory, speed and smoothness of the movement.

\subsection{PPI Testing Outcome}

The combined MaLT game was tested by the PPI group at two different stages of development. During the first testing, both $S$ and $B$ played the game and gave very positive feedback about the game. They especially liked that the game had text to speech that read the questions out aloud which made it easy for them to follow the game. $S$ and $B$ found the different tasks challenging but also enjoyed the game at the same time. They gave very positive response to the game based therapy and mentioned that they found it much more enjoyable using the Kinect and receiving instant feedback than when undergoing more traditional therapy exercises. One of their suggestions was to include speaking opportunities within the game as finding words presents one of the greatest challenges for their communication. This feature was subsequently included in the games.

\subsection{Results of the Pilot Study}

The three stroke survivors, IB, RB and $\mathrm{RH}$ who took part in the pilot engaged with the therapy for 8,6 and 6 weeks of therapy respectively. We looked at three measures of engagement and therapy structure. Number of trials completed during therapy, time spent playing the three structured therapy games (Single Word Comprehension, Initial Phoneme and Rhyming Word) and number of attempts at each question by game and level. Figure 3 shows the number of trials completed each week as patients engaged in therapy. The figure shows variable numbers of trials completed each week, with all patients showing a drop off during weeks 3-4, followed by a second period of greater intensity. This test on the pilot data was chosen to look at the engagement of the patient in the therapy and estimate how much therapy they completed each week. This test could be used by therapists to monitor the engagement of a patient and their therapy dosage in order to evaluate them against 
the target, and thus, assess the progress made by patient depending on the amount of therapy they completed. Figure 4 shows the time spent playing each game. For all patients, the most time was spent on the Rhyming Word game, followed by the Initial Phoneme and the Single Word Comprehension game. The estimate of time spent on each therapy game could be used to assess patient progress and monitor whether the patient is focusing on the required area of therapy, for example, word identification from phoneme or rhyming word. With the use of these results, therapists can ask patients to target play on the game/s that would be most beneficial to them.

Table 1 shows the number of attempts patients made at each question until they got the correct answer, broken down by therapy game level. Easier levels typically take 2 attempts, whilst harder levels can take up to 4 or 5 attempts. The majority of the trials were completed at levels $1-3$, with fewer trials completed at the very hardest levels (7-8).

Table 1: Number of attempts by therapy game level

\begin{tabular}{lllllllll}
\hline $\begin{array}{l}\text { Number } \\
\text { of } \\
\begin{array}{l}\text { attempts } \\
\text { until }\end{array}\end{array}$ & 1 & 2 & 3 & 4 & 5 & 6 & 7 & 8 \\
correct & & & & & & & & \\
1 & 687 & 553 & 468 & 345 & 242 & 196 & 276 & 235 \\
2 & 149 & 96 & 91 & 58 & 28 & 31 & 28 & 27 \\
3 & 1 & 3 & 59 & 31 & 22 & 11 & 11 & 9 \\
4 & 0 & 0 & 0 & 1 & 13 & 12 & 2 & 9 \\
5 & 0 & 0 & 0 & 0 & 0 & 0 & 2 & 5 \\
\hline
\end{tabular}

Data is number of trials completed, collapsed across all participants

This table gives a quantitative measure of a patient's performance. If the patient is taking multiple attempts to give a correct answer it means that the patient is having difficulty with that level. The therapist can then ask the patient to focus their attention on specific problem areas. Such a table also gives the therapist a tool to monitor patient progress and see whether over time a patient takes fewer attempts to answer the questions. It was also observed that all three patients came across most of the objects in the database during the course of their trials over the weeks. $\mathrm{RH}$ came across all 765 unique items in the database (100\%), RB came across 705 unique items (92.16\%) and IB came across 759 unique items (99.22\%). This ensures that the therapy tool provides opportunity to the patients to revise and relearn most of the objects included in the database. The therapist could thus tailor the database according to the patient's needs.

Table 2 shows the statistics of average time taken for answering the questions throughout the duration of the study. It could be observed that most of the times, the patients IB and $\mathrm{RH}$ used their affected hand to answer the question, getting more exercise to their affected arm. Patient RB has however used his unaffected arm to answer maximum questions. This suggests that the affected hand was not capable of performing the required movements. Also, the average response time for RB is greater than that of $\mathrm{IB}$ and $\mathrm{RH}$, in spite of using the unaffected hand indicating that RB has greater language impairment. Data in this table, when calculated at intervals during the course of therapy can show improvement in a patient's performance if their response time is decreasing. Also, it could be used to monitor if a patient is 
using their affected arm to answer the questions. Thus, these tests on the patient data could be used to monitor the engagement, therapy dosage as well as their performance over the time.

Table 2. Response time statistics. Average and Median response time to select correct answer across the duration of trial and its standard deviation as well as percentage of questions answered with affected hand.

\begin{tabular}{ccccc}
\hline Participants & $\begin{array}{c}\text { Average } \\
\text { Response } \\
\text { time }(\mathrm{sec})\end{array}$ & $\begin{array}{c}\text { Median } \\
\text { Response } \\
\text { time (sec) }\end{array}$ & $\begin{array}{c}\text { Standard } \\
\text { Deviation }\end{array}$ & $\begin{array}{c}\text { Affected } \\
\text { hand used } \\
\text { to select } \\
\text { objects }\end{array}$ \\
\hline IB & 6.00 & 5.09 & 3.65 & $90.06 \%$ \\
$\mathrm{RB}$ & 8.30 & 7.13 & 4.80 & $80.46 \%$ \\
$\mathrm{RH}$ & 6.28 & 5.09 & 3.92 & $90.27 \%$ \\
\hline
\end{tabular}




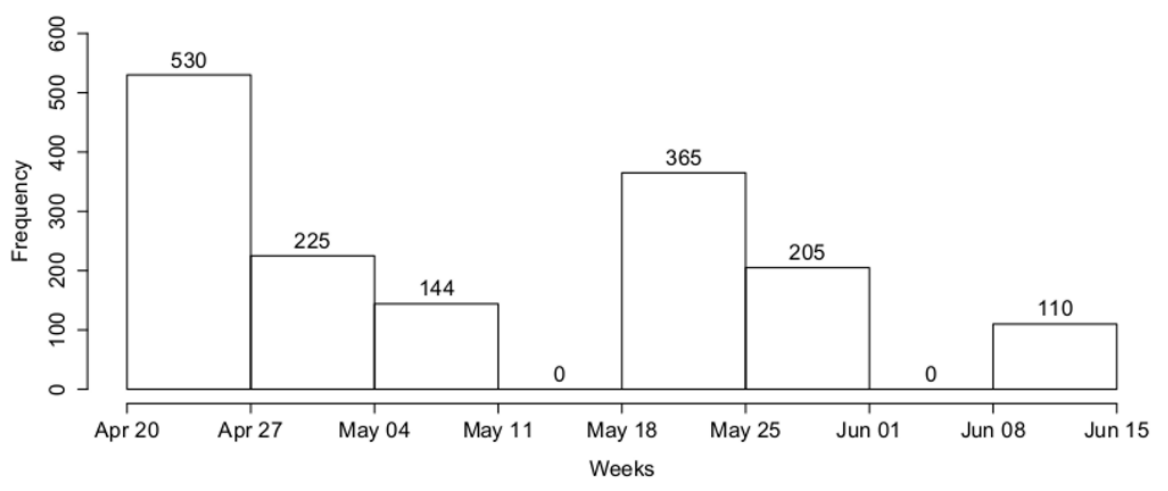

RB

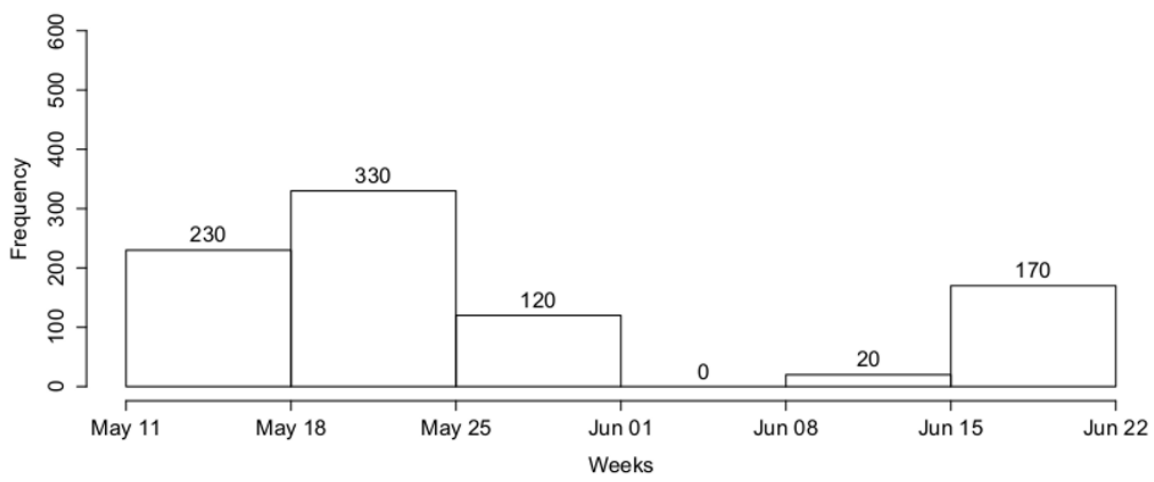

RH

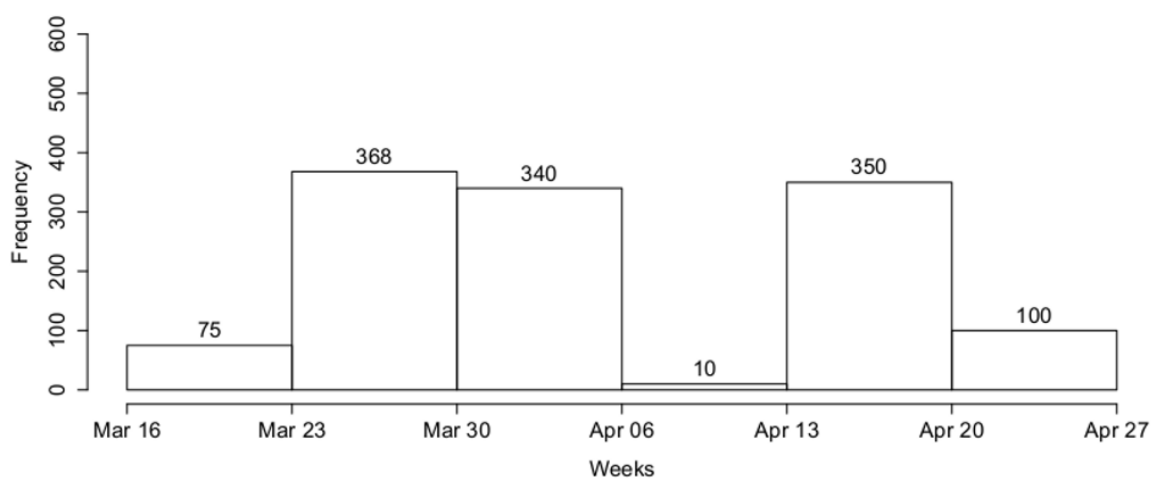

Figure 3: Total number of trials completed each week for all the three patients. 


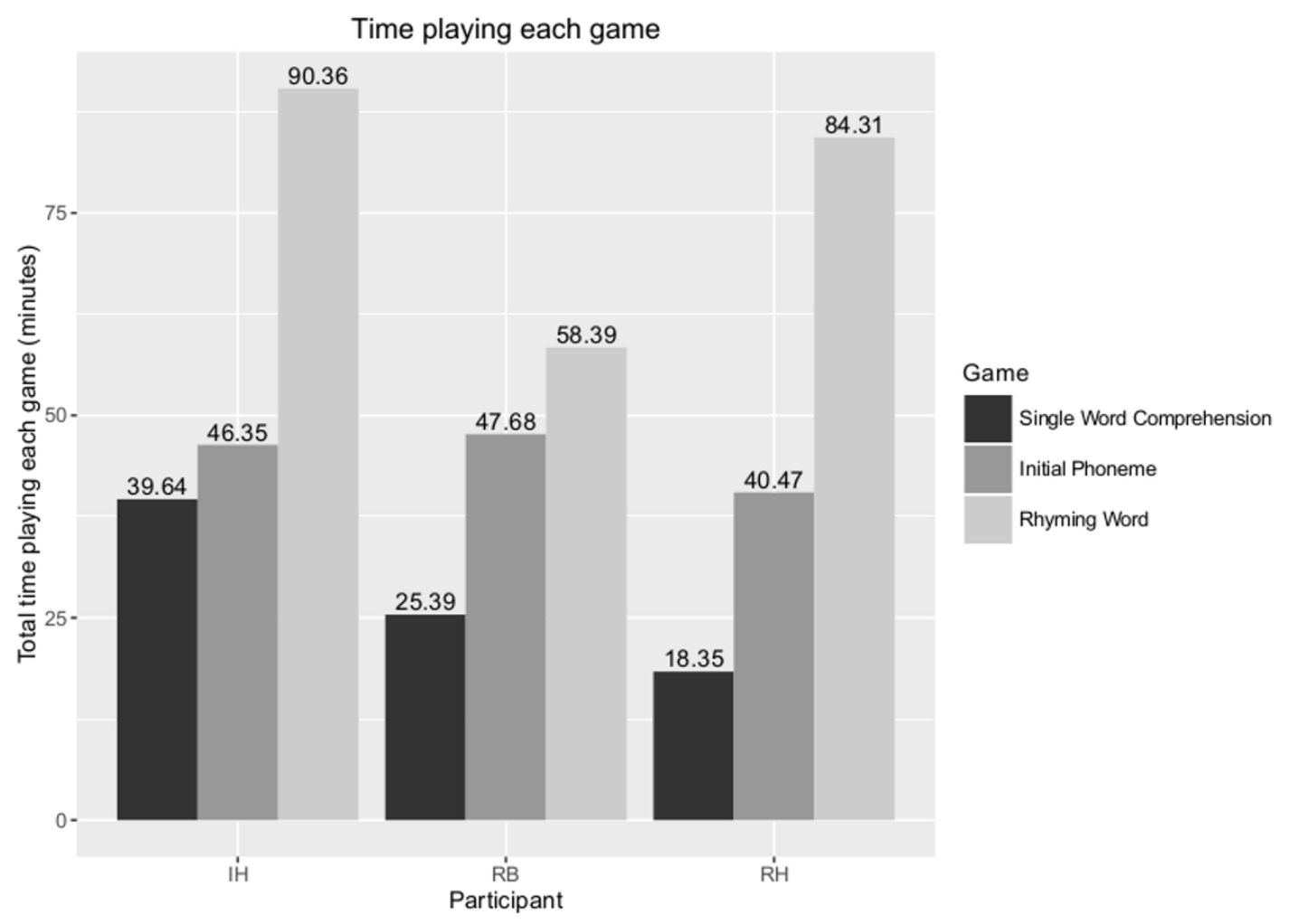

Figure 4: Overview of engagement in therapy. Total time spent on playing each game for all the patients over the entire duration of the pilot trial.

\subsection{Evaluation of the Achieved Key Requirements}

Provision of therapy: MaLT provides three different games incorporating different aspects of speech therapy including object recognition, phoneme identification, rhyme detection and an additional speaking task. Each of the three games has 8 difficulty levels.

Usability: MaLT is intuitive and does not need special instructions. This has been demonstrated by the amount of time patients played the games in the pilot study after only having one short instruction sessions (Figure 3 and Figure 4).

Engaging: Patients found MaLT engaging as they completed the trials on their own without supervision, this shows that they were motivated to play the game. The plots of time spent playing, distribution of play across the different games also suggest that patients found it engaging. A formal assessment of the feedback from the patients will be done in the future.

Feedback: Easy to comprehend feedback was provided as a simple numerical score with an emoticon reflecting their performance at the end of each level. 
Monitoring: A data visualisation tool was developed separately that allowed aggregation of the data recorded from the patients to monitor their performance. It was used to extract Figure 3, Figure 4, Table 1 and Table 2. Data within a session and across different sessions can be monitored.

Flexibility: Design of MaLT provides options to vary gameplay which can be done by therapists without the need for any reprogramming of the system by simply altering the database of objects to suit the patient needs.

Reporting: The MaLT system records all the patient's progress data automatically as detailed in section 4.1 MaLT Data Recording. The data is recorded as separate XML files per day and the visualization tool can aggregate the data together and present it as a table and different plots.

Low cost and home environment: MaLT only needs a Kinect sensor and can be run on any windows PC with USB 3.0.

Playability: Playability of the MaLT system can be seen from the successful pilots with very little training given to, or required by the patients. The patients found the game motivating and engaging as seen from their results for time spent playing the game. MaLT seemed to be an effective way of providing therapy and facilitating learning and language comprehension through gamification which also gives satisfaction to the patients via self-initiated home based therapy.

\section{Discussion}

MaLT has successfully combined motor and linguistic aspects of rehabilitation for acquired brain injury (ABI). To our knowledge, this is the first example of a multidisciplinary tool targeting upper limb and phonological awareness/language rehabilitation. By combining exercises for frequently co-morbid impairments, it is hoped that MaLT may provide a time efficient rehabilitation tool.

A core motivation for MaLT is the pressing need to provide an evidence-based dosage of impairment based rehabilitation services. Current evidence indicates that a minimum of 100 hours of therapy input is required to effect clinically significant changes [11]. In a 1:1 model it is estimated that 100 hours of therapy would cost, on average, £7800 for speech and language therapy and £9300 for physiotherapy [27]. All patients engaged with therapy for at least 6 weeks once started, without any further human support or guidance. Each patient completed approximately 300 trials in a typical week. The total reaction time for 300 trials was about 2 hours, although the time engaging with the game was considerably more which includes the speech task as well. This is less time than we were anticipating, and it may be that periodic monitoring or a diary is needed to ensure that individuals complete therapy activities each day as well as identification of the calendar duration and intensity of the therapy by conducting larger scale studies is necessary. Individuals showed a clear preference for particular games (the Rhyming Word). We are currently collecting user feedback to explore why these preferences arise. While the provision of sufficient therapy content is crucial, therapy must be targeted appropriately and change in difficulty as the individual progresses. Our manipulation of level was valid, showing more attempts needed and fewer trials completed as level difficulty increased. Therapy must be sufficiently engaging and motivating to ensure uptake by the patient. On its own, rehabilitation delivered via the Kinect motion sensor has been found to be motivating for $\mathrm{ABI}$ patients $[28,29]$. By involving service users in the 
design process, we have increased motivational aspects of the therapy and usability. However, further development work will enhance gamification aspects [30] which can further enhance extrinsic motivation for therapy uptake [31] and potentially increase time spent on each game and equalise the time playing each game.

Collaborative and social aspects of rehabilitation gaming and its implications on the game design have been identified by [32]. Stroke or any other illnesses often come with social isolation and hence it is useful for rehabilitation games to incorporate social aspects and provide opportunity for collaboration to the patients. This could be commonly achieved by incorporating online gaming aspects [32]. MaLT is currently an offline therapy tool designed as such to keep the costs, dependencies and overheads low as it is used in the homes of the patients. Patients using MaLT can play it with their younger children/grandchildren as naming and identifying the objects could be interesting as well as educational to children. Thus, the current version of MaLT could be played with family members which can improve the communication and socialization of the patients with their family. In future, collaborative as well as competitive features could be added to MaLT so that patients can play it together either in clinical settings or online at their homes.

The main target audience of the MaLT system were brain injury patients as the project was funded specifically for brain injury therapies. However, the concept, structure and design of MaLT could allow it to be used by a wider audience. It could be used as an engaging therapy tool for children with learning disabilities including developmental reading and language impairments providing an easy to use home based educational language therapy tool. It could also be used as a general language learning tool by non-English speaking children and adults as it focuses on identification of objects, rhyming words and phonemes and speaks it out aloud to learn vocabulary and pronunciation.

A simpler version of MaLT could also be used by pre-schoolers to learn new words interactively.

By combining multiple therapy targets, extensive unique game-play content and aspects of gamification, we believe that MaLT can provide a costand time-efficient rehabilitation tool for individuals with $\mathrm{ABI}$. Future clinical trials will examine the efficacy of MaLT in chronic ABI survivors and further user-feedback will be used to influence future development.

\section{Future Directions}

Pilot work will be enhanced to examine efficacy of MaLT. We will explore whether engagement with MaLT enhances phonological awareness, gross upper limb movement, single word comprehension and word retrieval in a case series of patients with $\mathrm{ABI}$. Based on results of pilot efficacy work obtained and further user feedback, future work will aim to enhance and extend the therapy programme and test efficacy in a large scale randomised controlled trial. The optimum duration and intensity of the therapy would be identified. Larger scale trials can also help to identify whether MaLT therapy is more effective in shorter duration of engagement as compared to the traditional therapy. Additional different set of games is also being developed.

Work is underway to integrate the visualization tool of the data for therapists within the MaLT system. A web based visualization tool will be made available for examining the patient's performance data. The success of initial trials on stroke survivors has led to collaboration with a company with the intention of 
commercializing the MaLT combined therapy approach.

\section{Conclusions}

Motion sensor technology can be employed to target multiple cognitive functions simultaneously. Patient user members of the research team indicate satisfaction with this multimodal approach to rehabilitation techniques and enhanced usability for individuals with acquired brain injury. This approach has the capacity to provide high dosage therapy in the home environment to a significant number of individuals living with the long-term consequences of $\mathrm{ABI}$. The initial studies on stroke survivors have demonstrated that the combined therapy approach is viable and that the stroke survivors with chronic language impairments can use the software independently. The outputs of this study will inform planned larger scale future trials in order to assess the effectiveness of the therapy tasks on language and motor performance. The patients of the study gave a very positive feedback and reported MaLT to be engaging and easy to use for home based therapy.

\section{Acknowledgment}

Our thanks to our trial participants, PPI and Headway Brain Injury Association for their contributions to the game and their support for the project. Thanks are due to Emma Pilkington for set up and training of the participants for the trials and Kaden Zhou for developing the visualization toolkit.

\section{References}

1. State of the Nation Stroke statistics - January 2016. Stroke Association; 2016.

2. Lawrence E, Coshall C, Dundas R, Stewart J, Rudd A, Howard R, et al. Estimates of the Prevalence of Acute Stroke Impairments and Disability in a Multiethnic Population. Stroke. 2001; 32(6): 1279-1284.

3. Hiller SL, Sharpe MH. Outcomes 5 years post traumatic brain injury (with further reference to neurophysical impairment and disability). Brain Injury. 1997; 11(9): 661675.

4. Engelter S, Gostynski M, Papa S, Frei M, Born C, Ajdacic-Gross V, et al. Epidemiology of Aphasia Attributable to First Ischemic Stroke: Incidence, Severity, Fluency, Etiology, and Thrombolysis. Stroke. 2006; 37(6): 1379-1384.

5. M. Gil, M. Cohen, C. Korn, Z. Grosw. Vocational outcome of aphasic patients following severe traumatic brain injury. Brain Injury. 1996; 10(1): 39-46.

6. Nichols-Larsen D, Clark P, Zeringue A, Greenspan A, Blanton S. Factors Influencing Stroke Survivors' Quality of Life During Subacute Recovery. Stroke. 2005; 36(7): 1480-1484.

7. Kauhanen M-L, Korpelainen J, Hiltunen P, Määttä R, Mononen H, Brusin E, et al. Aphasia, Depression, and Non-Verbal Cognitive Impairment in Ischaemic Stroke. 
Cerebrovasc Dis. 2000; 10(6): 455-461.

8. Hilari K, Northcott S, Roy P, Marshall J, Wiggins RD, Chataway J, et al. Psychological distress after stroke and aphasia: the first six months. Clinical

Rehabilitation. 2010; 24(2): 181-190. 9. Picelli A, Tamburin S, Gajofatto F, Zanette G,

Praitano M, Saltuari L, et al. Association between Severe Upper Limb Spasticity and Brain Lesion Location in Stroke Patients. BioMed Research International. 2014; 2014: 1-6.

10. Geva S, Baron J-C, Jones PS, Price CJ, Warburton EA. A comparison of VLSM and VBM in a cohort of patients with post-stroke aphasia. Neurolmage: Clinical. 2012; 1(1): 37-47.

11. Heinsius T, Bogousslavsky J, Van Melle G. Large infarcts in the middle cerebral artery territory: Etiology and outcome patterns. Neurology. 1998; 50(6): 1940-1943.

12. Meister IG, Boroojerdi B, Foltys H, Sparing R, Huber W, Töpper R. Motor cortex hand area and speech: implications for the development of language.

Neuropsychologia. 2003; 41(4): 401-406.

13. Meister IG, Sparing R, Foltys H, Gebert D, Huber W, Töpper R, et al. Functional connectivity between cortical hand motor and language areas during recovery from aphasia. Journal of the Neurological Sciences. 2006; 247(2): 165-168.

14. Cicerone KD, Dahlberg C, Malec JF, Langenbahn DM, Felicetti T, Kneipp S, et al. Evidence-Based Cognitive Rehabilitation: Updated Review of the Literature From 1998 Through 2002. Archives of Physical Medicine and Rehabilitation. 2005 ;86(8): 1681-1692.

15. Hinckley JJ, Craig HK. Influence of rate of treatment on the naming abilities of adults with chronic aphasia. Aphasiology. 1998; 12(11): 989-1006.

16. Platz T, Winter T, Müller N, Pinkowski C, Eickhof C, Mauritz K-H. Arm ability training for stroke and traumatic brain injury patients with mild arm paresis: A singleblind, randomized, controlled trial. Archives of Physical Medicine and Rehabilitation. 2001; 82(7): 961-968.

17. Meinzer M, Breitenstein C, Westerhoff U, Sommer J, Rosser N, Rodriguez AD, et al. Motor Cortex Preactivation by Standing Facilitates Word Retrieval in Aphasia. Neurorehabilitation and Neural Repair. 2010; 25(2): 178-187.

18. Bhogal SK, Teasell R, Speechley M, Albert ML. Intensity of Aphasia Therapy, Impact on Recovery - Aphasia Therapy Works! Stroke. 2003; 34(4): 987-993.

19. Bowen A, Hesketh A, Patchick E, Young A, Davies L, Vail A, et al. Effectiveness of enhanced communication therapy in the first four months after stroke for aphasia and dysarthria: a randomised controlled trial. BMJ. 2012; 345: e4407.

20. Chang Y-J, Chen S-F, Huang J-D. A Kinect-based system for physical rehabilitation: A pilot study for young adults with motor disabilities. Research in Developmental Disabilities. 2011; 32(6): 2566-2570. 
21. Lange B, Chang C-Y, Suma E, Newman B, Rizzo AS, Bolas M. Development and evaluation of low cost game-based balance rehabilitation tool using the microsoft kinect sensor. In: 2011 Annual International Conference of the IEEE Engineering in Medicine and Biology Society; 2011. p. 1831-1834.

22. Lloréns R, Albiol S, Gil-Gómez J-A, Alcañiz M, Colomer C, Noé E. Balance rehabilitation using custom-made Wii Balance Board exercises: clinical effectiveness and maintenance of gains in an acquired brain injury population. International Journal on Disability and Human Development. 2014; 13(3): 327-332.

23. Simmons S, Mccrindle R, Sperrin M, Smith A. Prescription Software for Recovery and Rehabilitation Using Microsoft Kinect. In: Proceedings of the 7th International Conference on Pervasive Computing Technologies for Healthcare; 2013. p. 323326.

24.Microsoft Windows Text-to-Speech for Unity, Assistant to the Lounge-About [Internet]. Chadweisshaar.com. c2015 [cited 2016 July 18]. Available from: http://www.chadweisshaar.com/blog/2015/ 07/02/microsoft-speech-for-unity/.

25.Greenwood A, Grassly J, Hickin J, Best W. Phonological and orthographic cueing therapy: A case of generalised improvement. Aphasiology. 2010; 24(9): 991-1016.

26. Lohse K, Shirzad N, Verster A, Hodges N, Van Der Loos, HF. Video Games and Rehabilitation. Journal of Neurologic Physical Therapy. 2013; 37(4): 166-175.

27. Curtis L. Unit Costs of Health and Social Care 2011. Kent: Personal Social Services Research Unit; 2011.

28. Broeren J, Bellner AL, Fogelberg M, Goransson O, Goude D, Johansson B, et al. Exploration of computer games in rehabilitation for brain damage. In: The 7th International Conference on Disability, Virtual Reality and Associated Technologies with ArtAbilitation; 2008. p. 75-80.

29. Chang YJ, Chen SF, Huang JD. A Kinect-based system for physical rehabilitation: A pilot study for young adults with motor disabilities. Res Dev Disabil. 2011; 32(6): 2566-2570.

30. Ferreira C, Guimarães V, Santos A, Sousa I. Gamification of Stroke Rehabilitation Exercises Using a Smartphone. In: Proceedings of the 8th International Conference on Pervasive Computing Technologies for Healthcare; 2014. p. 282-285.

31. Charles D, McDonough S. A participatory design framework for the gamification of rehabilitation systems. In: Proceedings of the 10th International Conference on Disability, Virtual Reality \& Associated Technologies; 2014. p. 293-296.

32. González-González C, Toledo-Delgado P, Collazos-Ordoñez C, GonzálezSánchez JL. Design and analysis of collaborative interactions in social educational videogames. Computers in Human Behaviour. 2014; 31: 602-611. 